\title{
Angewandter Strahlenschutz in der Neonatologie
}

\section{Einleitung}

Die Strahlenempfindlichkeit von Neugeborenen und Säuglingen ist weitestgehend unbekannt, dürfte jedoch um ein Mehrfaches höher einzuschätzen sein als die des Erwachsenen. Andererseits wird ein Großteil der konventionellen Röntgenuntersuchungen bei Neugeborenen und Säuglingen angefertigt. Da sich Kinder, und hier vor allem Neugeborene und Säuglinge, bezüglich Anthropometrie,
Anatomie, Physiologie, Psychologie und Strahlenbiologie deutlich von Erwachsenen unterscheiden, sind an den Strahlenschutz in der Neonatologie besondere Anforderungen zu stellen.

\section{Strahlenqualität}

Bei gegebener Bildempfängerdosis bestimmt die Anthropometrie des Patienten über den Durchstrahlungsdurchmesser die optimale Strahlenqualität und damit die Durchdringungsfähigkeit der Röntgenstrahlung, welche dem geringen Durchstrahlungsdurchmesser bei Neugeborenen und Säuglingen anzupassen ist. Die Strahlenqualität wird durch Röhrenspannung und Gesamtfilterung definiert. In den Leitlinien der Deutschen Bundesärztekammer wird für Thorax-Radiografien bei Neugeborenen eine Röhrenspannung von 60 - 80 kV empfohlen, im angloamerikanischen Raum werden hier sogar Röhren- 
spannungen von 50-60 kV angewandt. Aufgrund der sonst niedrigeren Kontrastauflösung ist die Anwendung höherer Röhrenspannungen in der Neonatologie nicht sinnvoll, außerdem könnte dann die minimale Schaltzeit des Röntgengenerators unterschritten werden. Die in den Leitlinien empfohlene Gesamtfilterung von 3,5 mm Al + 0,1 mm Cu kann vor allem bei modernen Röntgengeräten mit fest eingestellter Gesamtfilterung nicht mehr immer verwirklicht werden.

\section{Dosisbedarf}

Aufgrund des geringen Durchstrahlungsdurchmessers ist der Dosisbedarf bei neonatologischen Röntgenaufnahmen sehr gering, sodass sowohl die Messkammer zur Messung des Dosisflächenprodukts als auch die fakultativ zur Anwendung gelangende Messkammer zur Messung der Austrittsdosis zur korrekten Messung niedriger Strahlendosen in der Lage sein muss.

\section{Einblendung}

Die korrekte Einblendung des Strahlenfeldes ist bei Neugeborenen und Säuglingen aufgrund deren geringer Körperabmessungen vergleichsweise schwierig und stellt insbesondere bei Inkubator-Aufnahmen oftmals eine Herausforderung für den Untersucher dar.

\section{Belichtungsdauer}

Zur Verminderung von Bewegungsartefakten durch Atemexkursionen und Herzaktivität sind äußerst kurze Belichtungsdauern erforderlich. Bei neonatologischen Thorax-Aufnahmen sollte eine Grenzbe- lichtungsdauer von 4 ms nicht überschritten werden. Generell sollte bei pädiatrisch genutzten Röntgengeräten eine Möglichkeit zur Einstellung bzw. Ablesung der Belichtungsdauer vorgesehen sein; dies ist insbesondere bei fahrbaren Geräten oftmals nicht der Fall.

\section{Strahlengang}

Der Strahlengang bestimmt ganz wesentlich die relative Exposition der in unterschiedlichen Tiefenlagen unter der Hautoberfläche lokalisierten Organe und Gewebe; so ist beim antero-posterioren Strahlengang die relative Strahlenexposition oberflächennaher Organe wie der Schilddrüse und der Mammae deutlich höher als in postero-anteriorer Strahlrichtung. Allerdings wird eine Röntgenaufnahme bei Neugeborenen und Säuglingen in der Regel im Liegen oder im Hängen und unter Beobachtung der Atemexkursionen angefertigt und daher meist ein anteroposteriorer Strahlengang gewählt.

\section{Fokus-Haut-Abstand}

Bei freier Belichtung mit fahrbaren Röntgengeräten bewirkt eine geringfügige Verringerung des Fokus-Haut-Abstands aufgrund des Abstandsquadratgesetzes eine deutliche Erhöhung der Einfalldosis, sofern das mAs-Produkt nicht entsprechend angepasst wird. Die Gefahr einer relativen Überexposition von Neugeborenen ist somit v. a. bei Inkubator-Aufnahmen besonders groß; so bewirkt eine Verringerung des Fokus-Haut-Abstandes um 1 bzw. $2 \%$ bzw. $10 \%$ eine Erhöhung der Einfalldosis um etwa 2 bzw. $4 \%$ bzw. $20 \%$.

\section{Schlussfolgerung}

Die Optimierung der Strahlenexposition des Patienten stellt vor dem Hintergrund einer vernünftigen Bildqualität einen multimodalen Prozess dar, in welchem alle technischen Komponenten der Röntgeneinrichtung unter Berücksichtigung der anthropometrischen Eigenschaften des Patienten aufeinander abgestimmt werden müssen. In diesem Zusammenhang ist von den Röntgengeräteherstellern nachdrücklich eine Weiterentwicklung spezifischer Röntgeneinrichtungen für die Pädiatrie zu fordern.

\section{Interessenkonflikt}

Die Autoren geben an, dass kein Interessenkonflikt besteht.

\section{Autorinnen/Autoren}

Michael Seidenbusch

Klinikum der Universität München, Kinderradiologie

Korrespondenzadresse

PD Dr. Michael Seidenbusch

Klinikum der Universität München

Kinderradiologie

Lindwurmstr. 4

80337 München

michael.seidenbusch@med.uni-

muenchen.de

Bibliografie

DOI https://doi.org/10.1055/a-0943-1526 Online-Publikation: 2019

Fortschr Röntgenstr 2019; 191: S126-S127

(c) Georg Thieme Verlag KG, Stuttgart · New York ISSN 1433-5972 